\begin{tabular}{|c|l|}
\hline Title & Juvenile hormone titre and related gene expression during the change of reproductive modes in the pea aphid \\
\hline Author(s) & $\begin{array}{l}\text { Ishikawa, A sano; Ogawa, Kota; Gotoh, Hiroki; Walsh, Thomas K.; Tagu, Denis; Brisson, Jennifer A.; Rispe, Claude; } \\
\text { Jaubert-Possamai, Stéphanie; Kanbe, Takashi; T subota, Takuy a; Shiotsuki, Takahiro; Miura, Toru }\end{array}$ \\
\hline Citation & $\begin{array}{l}\text { Insect Molecular Biology, 21(1), 49-60 } \\
\text { https://doi.org/40.1111j.1365-2583.2011.01111.x }\end{array}$ \\
\hline Issue Date & 2012-02 \\
\hline Doc URL & http://hdl.handle.net/2115/51745 \\
\hline Rights & The definitive version is available at http://onlinelibrary.wiley.com/ \\
\hline Type & article (author version) \\
\hline File Information & IMB21-1_49-60.pdf \\
\hline
\end{tabular}

Instructions for use 


\section{Juvenile hormone titer and related gene expression during the change of reproductive modes in the pea aphid}

Asano Ishikawa ${ }^{1 *}$, Kota Ogawa ${ }^{1^{*}}$, Hiroki Gotoh ${ }^{1}$, Thomas K. Walsh ${ }^{2}$, Denis Tagu ${ }^{3}$, Jennifer A. Brisson ${ }^{4}$, Claude Rispe ${ }^{3}$, Stéphanie Jaubert-Possamai ${ }^{3}$, Takashi Kanbe ${ }^{5}$, Takuya Tsubota ${ }^{6}$, Takahiro Shiotsuki ${ }^{6}$ and Toru Miura ${ }^{1}$

*This author equally contributed.

1 Graduate School of Environmental Science, Hokkaido University, Sapporo, Japan

2 CSIRO Ecosystem Sciences, Clunies Ross St, Acton, ACT, 2601, Australia

3 INRA UMR 1099 BiO3P 35000 Rennes, France

4 School of Biological Sciences, University of Nebraska, Lincoln, USA

5 Graduate School of Agriculture, Hokkaido University, Sapporo, Japan

6 National Institute of Agrobiological Sciences, Tsukuba, Japan

Email addresses:

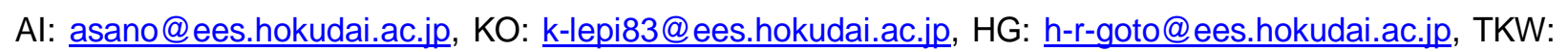
Tom.Walsh@csiro.au, DT: Denis.Tagu@rennes.inra.fr, JAB: 迆isson2@unl.edu, CR: Claude.Rispe@rennes.inra.fr, SJP: stephanie.jaubert@rennes.inra.fr, TK: $\underline{\text { t-kanbe@res.agr.hokudai.ac.jp, }}$ TT: tsubota@affrc.go.jp, TS: shiotsuk@affrc.go.jp, TM: miu@ees.hokudai.ac.jp

Send correspondence to:

Toru Miura, Ph.D.

Graduate School of Environmental Science, Hokkaido University, Sapporo, 060-0810, Japan email: miu@ees.hokudai.ac.jp

Tel \& Fax: $+81-11-706-4524$ 


\section{Abstract}

Most aphids show reproductive polyphenism, i.e. they alternate their reproductive modes from parthenogenesis to sexual reproduction in response to short photoperiod. Although juvenile hormone $(\mathrm{JH})$ has been considered a likely candidate for regulating the transition from asexual to sexual reproduction after photoperiod sensing, there are few studies investigating the direct relationship between $\mathrm{JH}$ titers and the reproductive mode change. In addition, the sequencing of the pea aphid genome now allows identification of the genes involved in the $\mathrm{JH}$ pathway which then allows us to examine their expression levels in relation to the reproductive-mode switch. Using LC-MS in the pea aphid, JHIII titer was shown to be lower in aphids producing sexual morphs under short-days than in aphids producing parthenogenetic morphs in long-days. The expression levels of genes upstream and downstream of $\mathrm{JH}$ action were quantified by real-time qRT-PCR across the reproductive mode change. The expression level of JH esterase $(\mathrm{JHE})$, which is responsible for $\mathrm{JH}$ degradation, was significantly higher in aphids reared under short-days. This suggests that the up-regulation of the $\mathrm{JH}$ degradation pathway may be responsible for the lower JHIII titer in aphids exposed to short-days, leading to the production of sexual morphs. (194/200 words) 


\section{Introduction}

In many organisms, responses to short-term local environmental changes are often accomplished via phenotypic plasticity, when a single genotype produces various phenotypes depending on the environment (Gilbert, 2001; West-Eberhard, 2003). Phenotypic plasticity producing discrete phenotypes from the same genotype is called polyphenism (Nijhout, 1999). Environmental heterogeneity favors plastic life-history strategies over non-plastic ones (Berrigan \& Scheiner, 2004). In temperate areas, organisms need to manage environmental heterogeneity in the form of seasonal changes in temperature, day length and host availability. Thus, they exhibit seasonal phenotypic changes such as changes in wing patterns in butterflies (Nijhout 1999) and reproductive-mode switches in daphnids (Eads et al., 2007) and aphids (Simon et al., 2002).

During the annual life cycles of most aphid species in temperate climates, several morphs are produced by clonal reproduction, illustrating seasonal polyphenism in these pest insects (Miyazaki, 1987; Dixon, 1998) (Fig. 1). From spring to summer, due to their high reproductive rate, aphids produce vast numbers of clonal offspring by viviparous parthenogenesis, resulting in large colonies that infest plants (Cuellar, 1977). At the beginning of fall, by responding to short photoperiod, clonal morphs give birth to sexual morphs (males and sexual oviparous females) that mate on their host plants. The sexual oviparous females lay overwintering eggs which hatch in the spring as parthenogenetic individuals that start a new cycle of clonal reproduction [reviewed in (Le Trionnaire et al. 2008)]. The single event of sexual reproduction per year increases genetic diversity, whereas the asexual events allow quick multiplication during the plant production seasons. Thus, the mechanisms regulating and underlying this switch between reproductive modes is critically important for aphids to appropriately fit their reproductive strategies to seasonally changing environments.

Long night-length is necessary and sufficient to trigger the switch from parthenogenesis to sexual reproduction in aphids and temperature changes modify this effect (Lees, 1973; Hardie, 1981). 
Several studies have suggested that the photoperiodic signal is detected by the protocerebrum in the brain of aphids through the cuticular head capsule (Lees, 1964; Lees, 1981). However, the putative photoperiodic receptors are still unknown (Gao et al., 1999). Early steps of photoperiod transduction involve a group of five neurosecretory cells located in the pars intercerebralis of the protocerebrum (Steel \& Lees, 1977), although the downstream mechanism of the photoperiodic signal is still largely unclear. Furthermore, the photoperiodic signal transduction mechanism is complicated by the trans-generational production of embryos due to viviparity since offspring generations develop as embryos within the grand-maternal and maternal ovaries.

Juvenile hormones $(\mathrm{JHs})$ are candidate molecules for regulating reproductive-mode switch after photoperiod sensing. JHs have been implicated in the production of alternative phenotypes (Nijhout, 1994; Nijhout, 2003; Hartfelder \& Emlen, 2005) in many cases of insect polyphenisms, such as the wing polyphenism in the cricket Gryllus rubens (Zera \& Denno, 1997), the phase polyphenism in locusts (Tanaka, 2001), the male dimorphism in several coleopterans (Emlen \& Nijhout, 1999), and the caste polyphenism in social insects (Nijhout, 1994; Nijhout \& Wheeler, 1982; Miura, 2005). Also, JH has been shown to regulate diapause in mosquitoes in response to photoperiod (Delinger, 2002). Therefore, several researchers have tested the hypothesis that $\mathrm{JH}$ could regulate the sexual polyphenism in aphids. For instance, although the corpus allatum (the organ that produces $\mathrm{JH}$ ) size and $\mathrm{JH}$ titer are not directly correlated, corpora allata volumes were different between long- and short-day reared vetch aphids Megoura viciae (Hardie, 1987). In addition, topical application of JH or juvenoids under short-days leads to the production of parthenogenetic females instead of sexual morphs in several aphid species (Lees, 1981; Mittler et al., 1976; Mittler et al., 1979; Corbitt \& Hardie, 1985; Hardie \& Lees, 1985a; Hardie \& Lees, 1985b). Finally, treatment with the anti-JH agent precocene induces male production in the green peach aphid Myzus persicae (Hales \& Mittler, 1983; Hales \& Mittler, 1988). However, these previous studies all investigated $\mathrm{JH}$ action indirectly; few studies have directly examined $\mathrm{JHIII}$, which is the only $\mathrm{JH}$ found in 
aphids, on sexual-morph production (Corbitt \& Hardie, 1985). One previous study used GC-MS to investigate the $\mathrm{JHIII}$ level difference between aphids reared under long- and short-day length in Megoura viciae (Hardie et al., 1985). However, because of the low sensitivity of the technique it was unclear whether JHIII titers correlated with day-length and asexual- or sexual-morph production. In addition, it has been also predicted that graded levels of $\mathrm{JH}$ would be responsible for the morph determination of embryos in maternal ovaries [reviewed in (Tagu et al. 2005)]. However, it is still unclear whether JH titer is different among aphids producing different morphs. The recent development of the LC-MS method to quantify JH titer in small insects (Westerlund \& Hoffmann, 2004; Schwartzberg et al., 2008) enables us to now investigate the relationship between $\mathrm{JH}$ titer and reproductive-mode change. In parallel, the recent sequencing and assembly of the pea aphid (Acyrthosiphon pisum) genome opens new routes to identify the genes encoding key components of $\mathrm{JH}$ synthesis and its downstream regulation, which then allows us to follow their expression during the process of reproductive-mode switch (International Aphid Genomics Consortium, 2010).

In this study, we carefully quantified JHIII titers in aphids producing different morphs under long and short-days. We show for the first time that pea aphids reared under short-day lengths have lower JHIII titer than those reared under long-day lengths. We also show that among the fourteen genes we measured, the steady-state transcript level for a predicted $\mathrm{JH}$ esterase 1 (JHE1), which is known to degrade $\mathrm{JH}$, was higher in aphids reared under short-day length. These results suggest that the lower JHIII titer observed in aphids exposed to short days can be correlated with a modification of the transcription of parts of the $\mathrm{JH}$ pathway. 


\section{Results}

\section{Production of sexual morphs}

Sexual morphs of the ApL strain of pea aphid Acyrthosiphon pisum were induced by rearing aphids at $15^{\circ} \mathrm{C}$ under short-days (8L16D). One parthenogenetic adult female produces a series of progeny and usually the different morphs (males, sexual females, parthenogenetic females) are produced sequentially. The detailed schedule of morph production differs from one strain to another. We thus first determined the sequence of morph production in the ApL strain. Parental parthenogenetic females (G1) reared under short days produced only parthenogenetic progeny during approximately eight days following the first larviposition (parturition of first-instar nymphs), and the production declined with time until the day 16 (Fig. 2A). Males began to be produced from day ten until the end of the larviposition period (Fig. 2A). Parthenogenetic females (G2) produced on day two were isolated and their progeny (G3) were followed (Fig. 2B). Sexual females were preferentially produced for approximately the first eight days, and then parthenogenetic females and males were produced (Fig. 2B). Under long-days (16L8D), only parthenogenetic females were consistently produced (data not shown). The detailed description of this progeny determination is illustrated in Fig. 3.

According to the progeny production schedules of strain ApL under short-days, we categorized the two periods of larviposition: early and late periods. Under the short-day condition, the reproductive switches (from parthenogenetic females to males in the SG1 progeny (G2 generation): Fig. 2A; and from oviparous sexual females to parthenogenetic females and males in the SG2 progeny (G3 generation): Fig. 2B) occurred around day eight. Periods earlier or later than day eight were designated the "early period" and "late period" respectively. Thus, it is expected that at day eight after the first larviposition almost all embryos contained in the ovaries of the G1 generation are future males, and almost all embryos in the ovaries of $\mathrm{G} 2$ aphids are parthenogenetic females. $\mathrm{JH}$ titers and gene expressions were examined in the following six aphid categories: Day1-LG1, Day8-LG1, Day1-SG1, Day8-SG1, Day1-SG2, and Day8-SG2 
(see Fig. 3 and below).

Day1-LG1 and Day8-LG1 represent parthenogenetic aphids reared under long-day lengths, just after or eight days after the first larviposition, respectively. In both, their ovaries contain embryos of future parthenogenetic females. These samples were used as the controls compared to short-day reared aphids. The other four categories were reared under short-day lengths (Day1-SG1, Day8-SG1, Day1-SG2, and Day8-SG2). Day1-SG1 and Day8-SG1 are groups of aphids of the first generation reared under short-day lengths. Day1-SG1 is a group of aphids just after the first larviposition having embryos of future parthenogenetic females, which have the potential to produce sexuals in the subsequent generation. Day8-SG1 is a group of aphids on day 8, whose ovaries contain embryos of future males. Day1-SG2 and Day8-SG2 are groups of aphids of the second generation reared under short-day lengths. Day1-SG2 is a group of aphids just after the first larviposition, whose ovaries contain embryos of future oviparous sexual females. Day8-SG2 is a group of aphids on day eight, containing embryos of future parthenogenetic females and males (See Fig. 2).

\section{JH titer determination}

To investigate the correlation between $\mathrm{JH}$ titer and reproductive modes, we compared $\mathrm{JH}$ titers among the six different aphid categories described above. Among various $\mathrm{JHs}$, JHIII is the only $\mathrm{JH}$ type found in A. pisum and LC-MS analyses we performed on the ApL strain supported this previous report (data not shown) (Schwartzberg et al., 2008). In comparison with aphids reared under long-day condition (Day1-LG1 and Day8-LG1), aphids reared under short-days (Day1-SG1, Day8-SG1, Day1-SG2, Day8-SG1) showed significantly lower JH titers (Fig. 4, Kruskal-Wallis test followed by the Peritz test, $\mathrm{p}<0.05)$. In addition, aphids also showed significant differences in $\mathrm{JH}$ titer between the early and late period of larviposition, although the difference was much smaller than the difference between day-length conditions. Among the six groups of aphids, JH III titer was the highest in Day8-LG1 aphids producing 
parthenogenetic females, and the lowest in Day8-SG1 aphids producing males. The JH titer in Day8-SG1 aphids is less than a third of that in Day8-LG1 aphids.

\section{Pea aphid orthologs of $\mathrm{JH}$ related genes}

The titer of $\mathrm{JH}$ in aphids producing different sexual morphs suggests that the $\mathrm{JH}$ level is kept low during the short-days for several days. Generally in insects, the hemolymphatic concentration of JH (i.e. $\mathrm{JH}$ titer) is regulated at various stages of $\mathrm{JH}$ accumulation: synthesis, sequestration, and degradation (Goodman \& Granger, 2005). In order to determine which of these regulatory processes might explain lower $\mathrm{JH}$ titers in the short-day reared aphids, we performed RNA expression analyses on factors involved in these steps. For this purpose, it was first necessary to identify pea aphid genes related to upstream or downstream pathways of $\mathrm{JH}$ action, referring to other insects species like Drosophila melanogaster and Tribolium castaneum as models. With the availability of the assembled pea aphid genome, we were able to search for homologous sequences by Blast and reciprocal Blast analyses (see Experimental Procedures). The JH-related genes identified in the pea aphid genome are listed in Table 1 and briefly described below.

\section{Genes involved in JH synthesis}

Allatostatin and allatotropin peptides are regulators of $\mathrm{JH}$ synthesis. These genes have recently been identified in the pea aphid (Huybrechts et al., 2010). In addition to these, we identified a putative allatostatin receptor (AstR) gene (Fig. S1: ACYPI008623). Alignment of the pea aphid sequence with insect homologs showed that the pea aphid $A s t R$ clearly falls into a clade of A-type AstR orthologs from other insects. Finally, four copies of $\mathrm{JH}$-acid methyltransferase (JHAMT) have recently been identified (Ollivier et al., 2010). 


\section{Genes involved in $\mathrm{JH}$ sequestration}

Hexamerins are important regulators of the hemolymphatic $\mathrm{JH}$ titer because they can bind to juvenoids to sequester and regulate $\mathrm{JH}$ titer (Zhou et al., 2007). Moreover, hexamerins have been linked to phenotypic plasticity (Zhou et al., 2007). Surprisingly, no hexamerin genes were found in the aphid genome database, although two prophenoloxidase (PPO) genes were found, which belong to the same protein familiy (Fig. S2).

\section{Genes involved in JH degradation}

We identified two potential Juvenile hormone esterase (JHE) genes (ACYPI001447, ACYPI007757), which were identified by their characteristic GQSAG motif (Ramsey et al., 2010). In addition, three potential juvenile hormone epoxide hydrolase (JHEH) genes were identified from the pea aphid genome. The group of epoxide hydrolases consists of two clusters (Arand et al., 1999). One cluster comprises soluble EHs (sEHs) from mammals, plants and bacteria, while the second cluster contains microsomal EHs (mEHs) from mammals and JHEH from insects. By constructing the phylogenetic tree of $\mathrm{mEHs}$ and sEHs from fungi, mammals and insects including pea aphid, it was shown that two of the aphid EHs (Fig. S3: ACYPI008135, ACYPI006263) were grouped with the sEHs, and only one EH clearly fell into the mEH clade with the JHEH genes from other insects (Fig. S3: ACYPI28868).

\section{Genes downstream of the $\mathrm{JH}$ pathway}

A number of genes have been implicated in the downstream effects of $\mathrm{JH}$ and copies of almost all of them were found in the pea aphid genome suggesting that the downstream effects of $\mathrm{JH}$ are regulated in a similar manner to other insects. Phylogenetic analysis of these genes showed that all of them grouped with their orthologs from other insect species. A methoprene-tolerant (Met) gene (Fig. S4: ACYPI31575), a FKBP39 gene (Fig. S5: ACYPI003035), a Chd64 gene (Fig. S6: ACYPI003572), two 
Ishikawa et al.

JH-related genes

Krüppel homolog 1 (Kr-h1) genes (Fig. S7: ACYPI000598, ACYPI006236), and a Broad gene (Fig. S8: ACYPI008576) were all identified. In addition, an ecdysone receptor $(E c R)$ gene and a retinoid $X$ receptor $(R X R)$ were recently identified in the pea aphid genome (Christiaens et al., 2010). EcR in the pea aphid shows two splice variants as in other insects (NM_001159360, NM_001159359, Watanabe et al., 2010)

\section{Expression profiles of $\mathrm{JH}$-related genes}

We quantified the relative expression levels of the $\mathrm{JH}$-related genes found in the six aphid categories producing different aphid morphs (Day1-LG1 and Day8-LG1 which produce parthenogenetic viviparous females, Day1-SG1 and Day8-SG1 which produce parthenogenetic viviparous females and males and Day1-SG2 and Day8-SG2 which produce parthenogenetic viviparous females, oviparous females, and males).

We first examined genes involved in JH synthesis, Allatotropin, AstR and JHAMT (Goodman \& Granger, 2005). Allatotropin expression was lower in Day1-LG1 and higher in Day8-SG1 and Day8-SG2 (Fig. 5A). AstR was highly expressed at day 8 after the first larviposition, in all groups of aphids (Fig. 5B). JHAMT did not show significant differences in gene expression among the six groups (Fig. $5 \mathrm{C})$. Although JHAMT4 tended to be expressed at lower levels in the second generation under short-days (SG2s), there was no significant difference (Fig. 5D). Among the genes involved in $\mathrm{JH}$ degradation, JHE1 and JHE2 were highly expressed in the first generation reared under short-day lengths (SG1s), and they were down-regulated in Day8-SG2 (Fig. 5E, 5F). In addition, JHE1 showed a significant negative correlation with JHIII titers ( $r s=-0.89, p<0.05$, Fig. 6). JHEH had lower levels of expression in aphids under long-days, and increased expression in Day1-SG2 aphids (Fig. 5G).

In genes downstream in the $\mathrm{JH}$ pathway, there was no overall pattern in the expression profiles. The expression of FKBP39 dropped gradually through generations under short-days (Fig. 5l). Chd64 was highly expressed in Day8-SG1, and Day8-SG2 (Fig. 5J). RXR was less expressed in Day8-SG1, and 
Ishikawa et al.

$\mathrm{JH}$-related genes

highly expressed in Day8-LG1 and Day8-SG2 (Fig. 5L). Broad showed an increase in expression on day 8 after the first larviposition, regardless of the morphs produced (Fig. 5M). The $K r-h 1$ expression was lower in Day1-SG2, and slightly higher in Day8-SG1 and Day8-SG2 (Fig. 5N). The expression patterns of Met and EcR were not significantly different among the 6 groups of aphids (Fig. $5 \mathrm{H}$ and $5 \mathrm{~K}$ ). 


\section{Discussion}

As shown in many cases of insect polyphenism, juvenile hormones play critical roles in developmental regulation. In this study, we showed that $\mathrm{JH}$ titer was correlated with the reproductive mode change between sexual and asexual reproduction in the pea aphid. Furthermore, by means of qRT-PCR, we searched for genes related to JH action, which were up- or down-regulated in association with the reproductive mode change.

In aphids, the control of sexual morph production by photoperiod has been observed by several groups (Marcovitch, 1924; Lees, 1959). The measurements of JH titer in this study showed that in the pea aphid adult female aphids reared under short-day lengths had markedly lower JHIII titer than aphids reared under long-days. As measurements were performed on whole body extracts, we did not track the hemolymphatic JHIII titer. In some aphid species, ectopic applications of JHs or JH analogues to aphids under short-day lengths induces the production of parthenogenetic females, instead of oviparous females (Hardie, 1981; Mittler et al., 1979; Corbitt \& Hardie, 1985; Hardie \& Lees, 1985a; Hardie \& Lees, 1985b). In addition, topical application of Kinoprene, one of the $\mathrm{JH}$ analogs, prevented photoperiod-induced male production in the green peach aphid Myzus persicae (Mittler et al., 1979). These previous studies suggest that a high endogenous $\mathrm{JH}$ titer influences the embryo to develop as a parthenogenetic female. Together with the JHIII measurements, it is suggested that, in the pea aphid, short-day lengths induce low JHIII titer resulting in the production of sexual morphs, and long-day lengths cause high JHIII titer leading to the production of parthenogenetic females. Temperature modulates the photoperiodic response inducing the reproductive mode changes (Lees, 1959; Le Trionnaire et al., 2008). For example, in the vetch aphid Megoura viciae, high-temperature treatments tended to shift the reproductive mode to parthenogenetic reproduction (Lees, 1963; Lees, 1966). The similar modulation of photoperiodism by temperature was reported in other aphid species including the pea aphid [reviewed in (Lees, 1966)]. LC-MS/MS analysis showed that $\mathrm{JHIII}$ titer in aphids reared at $25^{\circ} \mathrm{C}$ was about twice that of 
aphids reared at $10^{\circ} \mathrm{C}$ (Chen et al., 2007). Furthermore, we showed that JHIII titers in aphids reared in long-day conditions were more than two times higher than those of aphids under short-days. Taken together, these results suggest that the modulation of the photoperiodic response by temperature is accomplished by altering the basal level of JHIII titers. Thus, we hypothesize that JHIII integrates information about photoperiod and temperature, resulting in the switch to sexual reproduction at the appropriate time in the fall.

Besides $\mathrm{JH}$, other unknown factors might be involved with reproductive-mode changes, especially in the determination of embryonic fate, as suggested by the following observations. Although aphids producing males had the lowest JHIII titers, parthenogenetic females and males were produced simultaneously from the same mother in the late period of the second generation reared under short-day length (SG2). It is also possible that the heterogeneity of $\mathrm{JH}$ titer inside an aphid body may differentially affect embryos at different positions in the ovaries (Hardie, 1987). In the case of host-alternating species of Aphididae, parthenogenetic females on the secondary host produce winged males and gynoparae, both of which fly to the primary host, where the gynoparae deposit sexual females (Miyazaki, 1987). In that case, $\mathrm{JH}$ might affect the production of both of the migrants (male and gynoparae) in fall, and other factors may determine whether they develop to male or gynoparae. In addition, JHIII titers in aphids producing different morphs (Day1-SG1 producing parthenogenetic female and Day1-SG2 producing oviparous female) did not show significant differences. Since a circadian rhythm of JH titer was reported in the cricket Gryllus firmus, JH titer in the pea aphid might show the circadian rhythm (Zhao \& Zera, 2004; Zhao \& Zera, 2009). Thus, it is possible that the difference of $\mathrm{JH}$ titer among aphids producing different morphs might be detectable in other sampling times during a day. While the males are determined very early in the embryogenesis by the loss of one sex chromosome at the first maturation division of the oocyte, the determination of parthenotenetic and sexual females occurs later (Blackman, 1983). Thus, further studies by careful JH measurements will decipher the $\mathrm{JH}$ roles in determination of 
embryonic fates.

Genes related to the $\mathrm{JH}$ pathway were identified from the pea aphid genome by comparing gene models with orthologous genes found in other insects (Table 1). We found almost all genes that we surveyed in the pea aphid genome, although no putative hexamerins, which sequester $\mathrm{JH}$ in fat bodies, were found among the predicted genes, genomic scaffolds and ESTs. This may suggest that, instead of hexamerins, other factors are at play in the sequestration process. An alternative explanation could be that aphid hexamerin genes have extremely divergent sequences that prevented recognition; given the length and usually relatively high conservation for these genes, this seems less likely. The JH pathway regulates multiple aspects of development, metamorphosis, and reproduction in insects, so that genes involved in this pathway are conserved in the aphid genome. For some genes (JHAMT, JHE, Kr-h1), multiple gene copies were found. Since it is difficult to determine which of these are the functional orthologs, it will be necessary to perform functional assays in future analyses.

In order to search for $\mathrm{JH}$-regulating factors responsible for lowering $\mathrm{JH}$ titer in response to the decrease of photoperiod, the expression levels of genes involved in $\mathrm{JH}$ synthesis and degradation were quantified by real-time qRT-PCR. The expression profiles of Allatotropin, which stimulates JH synthesis in corpora allata did not correlate with $\mathrm{JH}$ titer differences. Further, AstR, the receptor of allatostatin that inhibits the $\mathrm{JH}$ synthesis, did not show any significant correlation, either. Moreover, at the final step of $\mathrm{JH}$ synthesis, JHAMT converts farnesoic acid and $\mathrm{JH}$ acids into methylfarnesoate and $\mathrm{JH}$, respectively. The expression of JHAMT did not show significant differences among the six aphid categories reared in longor short-day conditions, suggesting that the inactivation of $\mathrm{JH}$ synthetic pathways may not be responsible for the low $\mathrm{JH}$ titers in short-day length. Furthermore, the upstream factors regulating $\mathrm{JH}$ titer that were investigated in this study showed no correlation with the measured $\mathrm{JH}$ titers, so that the other pathways controlling $\mathrm{JH}$ titer (e.g. sequestration or degradation) may be responsible. 
JHEs are known to play important roles in $\mathrm{JH}$ degradation, lowering $\mathrm{JH}$ titers in many insects [reviewed in (Gilbert et al., 2000)]. For instance, in the wing polymorphism of crickets, it has been strongly suggested that the reduced JHE activity elevates $\mathrm{JH}$ titers, specifying the development of the short-winged morph (Zera, 2003; Zera, 2006; Zera et al., 2007). Among the pea aphid gene models, multiple gene candidates for JHEs were found, and the expression levels for two of them (JHE1 and JHE2) were examined. Results showed that the expression levels of both JHEs were significantly higher in the short-day lengths although they returned in the late period of second generation when parthenogenic females were again produced. Furthermore, JHE1 expression was negatively and strongly correlated with JHIII titers. In addition, the expression levels of JHEH, which is also involved in the JH catabolism, increased gradually in response to short-day lengths, especially in Day1-SG2. However, the JHEH activity degrading JHIII was shown to be extremely low using recombinant JHEH proteins (Tsubota, personal communication). Therefore, JHEH is suggested to play a minor role in JHIII degradation. These results suggest that $J H E$ is a good candidate as a critical factor regulating $\mathrm{JH}$ titer in response to the decrease of photoperiod. Under these conditions, the high JHE expression would cause the degradation of $\mathrm{JHIII}$, leading to the low $\mathrm{JH}$ titer, resulting in the induction of the sexual-morph. In contrast, under long-days, low JHE expression would allow a higher JHIII titer, inducing the production of parthenogenetic females. To confirm this hypothesis, further studies are needed, such as the measurement of JHE activity in aphids, the enzyme activity assay of each JHE, and the clarification of the epistatic relationship between $\mathrm{JH}$ titer and JHE activity.

In addition to the control of $\mathrm{JH}$ titer, some factors transmitting the $\mathrm{JH}$ signals to the downstream pathways should be responsible for the fate determination of developing embryos. Therefore, we also analyzed the expression pattern of genes involved in $\mathrm{JH}$ reception and its downstream pathways. However, none of the examined gene candidates could explain the production patterns of three reproductive morphs (parthenogenetic, oviparous females and males), except that $K r$ - $h 1$ showed a low 
expression level at the time when oviparous females were produced (Fig. 4N). Since the FKBP39 expression pattern slightly corresponded to the pattern of JHIII titers, showing lower expression in aphids producing sexual female or males (Day8-SG1, Day1-SG2, and Day8-SG2), so that this gene expression might be involved in the $\mathrm{JH}$ action controlling the reproductive-mode changes. Although EcR expression did not exhibit significant difference among the 6 groups of aphids, the two splice variants were not distinguished in the analyses. Since in the holometabolous insects each EcR isoform shows distinct steroid-stimulated response and involved in the different developmental process, it is still possible that isoform-specific expressions are involved in the regulation of reproductive-mode change.

In this study, we used only RNA extracted from aphid whole bodies. To describe in more detail the regulation of $\mathrm{JH}$ for the determination of embryonic fates, further gene expression analysis using mRNA from specific tissues will be necessary. Although not examined in this study, recent transcriptomic analyses showed that two genes related to the insulin signaling pathway were significantly regulated in aphids under short-day length producing sexual morphs (Le Trionnaire et al., 2009). These findings suggest that cross talk between these endocrine pathways integrates external and internal information such as photoperiod, temperature, and age, and determine the embryonic fates within the maternal ovarioles.

This study strongly suggests that JHIII plays a role in the reproductive mode change from parthenogenesis to sexual reproduction in response to seasonal changes. In addition, we identified genes involved in the $\mathrm{JH}$ pathway from the pea aphid genome, and their expression profiles were elucidated by qRT-PCR. As a result, we suggest that JHE is the key regulator responsible for the decrease of JHIII titer in response to short-day condition. The detailed mechanisms of reproductive polyphenism, such as the transduction of photoperiodic signals to JHIII titers, or the determination of the embryonic fate, still remain unknown. The integration of sensitive measurements of hormones, and spatiotemporal expression analyses together with functional analyses of genes regulated by photoperiodism, which are up- and 
downstream of endocrine factors, will reveal a more complete picture of the molecular mechanism underlying reproductive polyphenism in aphids. 


\section{Experimental Procedures}

\section{Insects}

The ApL strain of pea aphid Acyrthosiphon pisum (referred to as Sap05Ms2 in (Kanbe \& Akimoto, 2009)) was collected in Japan and used in this study. Parthenogenetic aphid cultures were maintained in tubes (diameter: $2.5 \mathrm{~cm}$, height: $10 \mathrm{~cm}$ ) in which a bean seedling (Vicia faba) was placed on wet vermiculite under long-day lengths (16L8D, $\left.20^{\circ} \mathrm{C}\right)$ (Wilkinson \& Ishikawa, 2000). Preliminary observations showed that in the ApL strain, winged parthenogenetic females produced no males even under short-days. In order to investigate the relationship between $\mathrm{JH}$ titer and offspring morphs including males, we used only wingless parthenogenetic females for the following experiments. To be sure that only wingless morphs are produced, only one wingless adult female was kept on a $3 \mathrm{~cm}$ vetch seedling in each generation (Ishikawa et al., 2008).

\section{Induction of sexual morphs}

The production of sexual morphs can be induced in ApL by subjecting the aphids to short day lengths ( 8 hours light and 16 hours dark). First-instar aphids produced by a single wingless mother were isolated and reared on another seedling at $15^{\circ} \mathrm{C}$ under short-days (8L16D) or long-days (16L8D) until they became adult aphids. They are represented here by the first generation (G1). Those reared under long-days are called "LG1" and those reared under short-days are called "SG1". After the onset of larviposition, each adult aphid was transferred to a new seedling daily to confirm the sequence of sexual/asexual morphs in the next generation. This new generation, which is produced on the day after the first larviposition, is referred to G2 and dubbed "SG2" for short-days reared insects. The corresponding LG2 were discarded and not kept for further analyses. The progeny of these aphids were reared for several days until their morphs were confirmed (parthenogenetic female, sexual female or male). In the adult stage, parthenogenetic females and oviparous females can be distinguished by the 
appearances of ovaries; asexual females have parthenogenetic ovaries with a number of embryos, while sexual females possess gametic ovaries containing only haploid eggs (Blackman, 1983). Males are discriminated by their wings, small abdomen and male gonads. Sample categories (Day1-LG1, Day8-LG1, Day1-SG1, Day8-SG1, Day1-SG2, and Day8-SG2) were determined based on the production schedules of reproductive morphs (Fig. 3).

\section{JH quantification}

To investigate the correlation between $\mathrm{JH}$ titer and the reproductive-mode change, we quantified $\mathrm{JH}$ titers in aphids producing sexual or asexual aphids. Individual aphids stored at $-80^{\circ} \mathrm{C}$ were homogenized in $400 \mu \mathrm{l}$ of methanol with $30 \mathrm{ng}$ fenoxycarb (Wako, Osaka, Japan) as an internal standard. Five replicated samples (each replicate derived from 5 aphids) were prepared for each of the 6 categories (Day1-LG1, Day8-LG1, Day1-SG1, Day8-SG1, Day1-SG2, and Day8-SG2). The sample was transferred into a glass vial, and $100 \mu \mathrm{l}$ of $2 \% \mathrm{NaCl}$ was added. They were extracted three times with $300 \mu \mathrm{l}$ of hexane (Wako, Osaka, Japan). After adding hexane, the sample was vortexed vigorously, incubated for 5 min at room temperature, and centrifuged $(965 \mathrm{~g})$ for $5 \mathrm{~min}$. The hexane (upper) phase was collected in a new glass vial. The combined hexane extract $(900 \mu l)$ was dried completely under vacuum and dissolved in $30 \mu \mathrm{l}$ of acetonitrile. The sample was stored at $-20^{\circ} \mathrm{C}$ until analysis. Experimental details were modified from Chen et al., 2007.

To quantify juvenile hormone titers in aphids, the experimental methods of LC-MS were slightly modified based on Westerlund et al., 2004 and Cornette et al., 2008. Briefly, $5 \mu$ from each $30 \mu l$ extracted sample was separated on a 150×2 mm2 C18 reverse-phased column (YMC-Pack Pro C-18.5 um, YMC Co., Ltd., Kyoto, Japan) protected by a guard column (YMC-Pack Pro, sphere ODS, YMC Co., Ltd., Kyoto, Japan) with gradient elution of water/methanol (0-15 $\min 80-100 \%$ methanol, $15-20$ min 100\% methanol) at a flow rate of $0.2 \mathrm{ml} / \mathrm{min}$, utilizing an Agilent $1100 \mathrm{HPLC}$ system with autosampler. See 
Ishikawa et al.

JH-related genes

Cornette et al., 2008 for more detailed methods about the TOF-MS condition. To evaluate the significant expression differences among the categories, Peritz tests were performed after Kruskal-Wallis test.

\section{JH pathway genes annotation and ortholog identification}

To search for pea aphid orthologs of $\mathrm{JH}$-related genes, we used protein sequences of JH-related genes of Drosophila melanogaster in a Blast search querying pea aphid Refseq or Gnomon predicted proteins from version 1.0 of the $A$. pisum genome (http://www.aphidbase.com/). Hits with e-values less than 1e-20 were examined, although the majority of the hits were much higher. If one (or two) hit was clearly more significant than the others, it was chosen as the putative pea aphid ortholog of the Drosophila sequence. Then, we performed Blast analysis in NCBI (http://blast.ncbi.nlm.nih.gov/) with the pea aphid sequences. Based on the results of reciprocal Blast searches, we assigned orthologs to the pea aphid sequences. To further confirm the orthologs, we made multiple alignments of related genes including the orthologs from the other insects, and construct neighbor-joining similarity trees of protein sequences using CLUSTALX program (Larkin et al., 2007) (http://www.clustal.org/). It was bootstrapped 1000 times to estimate confidence in the nodes.

\section{RNA extraction and real-time quantitative PCR}

Total RNA was extracted from five individuals stored at $-80^{\circ} \mathrm{C}$ using SV Total Isolation System (Promega, Madison, WI). Three replicated samples (=15 individuals) were prepared for each of the six categories (Day1-LG1, Day8-LG1, Day1-SG1, Day8-SG1, Day1-SG2, and Day8-SG2). For each sample, $1 \mu \mathrm{g}$ of total RNA was reverse transcribed with the High Capacity cDNA Reverse Transcription Kit according to the manufacturer instructions (Applied Biosystems, Foster City, CA). Relative quantification of cDNAs was performed using a SYBR Green I chemistry system and sequence detection system ABI PRISM 7500 (Applied Biosystems). For determining endogenous control of constitutive expression, the 
Ishikawa et al.

JH-related genes

suitability of different putative reference genes was evaluated. The references considered were Glyceraldehyde-3-phosphate dehydrogenase (GAPDH), beta-actin, Elongation factor 1 alpha (EF1a), and Ribosomal protein L32 (rpL32) genes. Using the softwares geNorm (Vandesompele et al., 2002) and Normfinder (Andersen et al., 2004), we determined that the expression level of EF1a was the most stable among the candidate reference genes. Therefore, EF1a was used as the reference gene to quantify relative gene expressions of genes of interest. Primers for both target and endogenous control transcripts were designed using the Primer Express software (Applied Biosystems, Foster City, CA, USA). For all qRT-PCR experiments, the production of gene-specific products was checked by careful scrutiny of melting curves (conducted at the end of all qRT-PCR reactions). Data acquisitions and analyses were performed by ABI Prism 7500 software v2.0.1 (Applied Biosystems, Foster City, CA, USA). Baselines and thresholds for Ct were set automatically. The relative standard curve method was used for qRT-PCR quantification as described in User Bulletin 2 for the ABI Prism 7700 Sequence Detection System (Applied Biosystems).

For aphid JH-related genes that showed duplication or expansion of copy numbers (such as the JHAMT and the $K r-h 1$ genes), it was not possible to design specific primers for each gene homolog. Therefore, we designed the primers to cover conserved regions shared by the homologs. For JHAMT, we were not able to find a completely conserved region shared by the four copies showing sufficient length to design a common primer. Thus, we designed a specific primer set for JHAMT4, in addition to the common primer set for all JHAMTs, although the reverse primer is slightly different between JHAMT1-3 and JHAMT4 (See Table S1; two bases in the region for reverse primer is different between JHAMT1-3 and JHAMT4). The resultant melt curves showed a single PCR product for both primer sets indicating that the sum expression of all the four homologs was probably quantified. For EcR, we designed a primer set for the common region shared by two splice variants.

To evaluate the significant expression differences among the categories, Tukey's multiple 
comparison tests $(p<0.05)$ were performed after one-factor ANOVA. The correlation between the JHIII titers and the expression levels of $\mathrm{JH}$-related genes was evaluated by Spearman's rank correlation $(p<0.05)$ 


\section{Acknowledgments}

We are grateful to R. Cornette for his valuable comments on the study and assistance in various experiments. Thanks are also due to Naoki Irie for their valuable suggestions on the study, especially about statistics. This work was supported by Grants-in-Aid for Scientific Research (Nos. 18370007, 20033002, 20657004 and 21677001) from the Ministry of Education, Culture, Sports, Science and Technology of Japan, and partly by a Research Grant from Inamori Foundation. It was also supported in France by ANR -genoplante, Aphicibles program and the CSIRO OCE Postdoctoral program. 


\section{References}

Andersen, C.L., Jensen, J.L. and Orntoft, T.F. (2004) Normalization of real-time quantitative reverse transcription-PCR data: A model-based variance estimation approach to identify genes suited for normalization, applied to bladder and colon cancer data sets. Cancer Res 64: 5245-5250.

Aoki, S. (1977) Colophina clematis (Homoptera, Pemphigidae), an aphid species with 'soldiers'. Kontyu. 45: $276-282$.

Arand, M., Hemmer, H., Durk, H., Baratti, J., Archelas, A., Furstoss, R. and Oesch, F. (1999) Cloning and molecular characterization of a soluble epoxide hydrolase from Aspergillus niger that is related to mammalian microsomal epoxide hydrolase. Biochem J 344: 273-280.

Berrigan, D. and Scheiner, S.M. (2004) Modeling the evolution of phenotypic plasticity. In Phenotypic Plasticity : Functional and Conceptual Approaches. (Dewitt, T.J., and Scheiner, S.M., eds.), pp. 82-97. Oxford University Press, Oxford, UK.

Blackman, R.L. (1987) Reproduction, cytogenetics and development. In Aphids: Their Biology, Natural Enemies and Control (Minks, A.K., and Harrewijn, P., eds.), pp. 163-195. Elsevier, Amsterdam, NL.

Chen, Z., Linse, K.D., Taub-Montemayor, T.E. and Rankin, M.A. (2007) Comparison of radioimmunoassay and liquid chromatography tandem mass spectrometry for determination of juvenile hormone titers. Insect Biochem Mol Biol 37: 799-807.

Christiaens, O., Iga, M., Velarde, R.A., Rougé, P. and Smagghe, G. (2010) Halloween genes and nuclear receptors in ecdysteroid biosynthesis and signalling in the pea aphid. Insect Mol Biol 19: 187-200.

International Aphid Genome Consortium (2010) Genome Sequence of the Pea Aphid Acyrthosiphon pisum. PLoS Biol 8: e1000313.

Corbitt, T.S. and Hardie, J. (1985) Juvenile hormone effects on polymorphism in the pea aphid, Acyrthosiphon pisum. Entomol Exp App/38: 131-135.

Cornette, R., Gotoh, H., Koshikawa, S. and Miura, T. (2008) Juvenile hormone titers and caste 
Ishikawa et al.

JH-related genes

differentiation in the damp-wood termite Hodotermopsis sjostedti (Isoptera, Termopsidae). J Insect Physiol 54: 922-930.

Cuellar, O. (1977) Animal parthenogenesis. Science 197: 837-843.

Delingher, D.L. (2002) Regulation of diapause. Annu Rev Entomol 47: 93-122.

Dixon, A.F.G. (1998) Aphid Ecology. Chapman \& Hall, London, UK.

Eads, B.D., Colbourne, J.K., Bohuski, E. and Andrews, J. (2007) Profiling sex-biased gene expression during parthenogenetic reproduction in Daphnia pulex. BMC Genomics 8: 464.

Emlen, D.J. and Nijhout, H.F. (1999) Hormonal control of male horn length dimorphism in the dung beetle Onthophagus taurus (Coleoptera : Scarabaeidae). J Insect Physiol 45: 45-53.

Emlen, D.J. (2001) Costs and the diversification of exaggerated animal structures. Science 291: 1534-1536.

Gao, N., von Schantz, M., Foster, R.G. and Hardie, J. (1999) The putative brain photoperiodic photoreceptors in the vetch aphid, Megoura viciae. J Insect Physiol 45: 1011-1019.

Gilbert, L.I., Granger, N.A. and Roe, R.M. (2000) The juvenile hormones: historical facts and speculations on future research directions. Insect Biochem Mol Biol 30: 617-644.

Gilbert, S.F. (2001) Ecological developmental biology: Developmental biology meets the real world. Dev Biol 233: 1-12.

Goodman, W.G. and Granger, N.A. (2005) The juvenile hormones. In Comprehensive Molecular Insect Science. (Gilbert, L.I., latrou, K., and Gill, S.S., eds.), pp. 319-408. Elsevier, Oxford, UK.

Hales, D.F. and Mittler, T.E. (1983) Precocene causes male determination in the aphid Myzus persicae. J Insect Physiol 29: 819-823.

Hales, D.F. and Mittler, T.E. (1988) Male production by aphids prenatally treated with precocene: prevention by short-term kinoprene treatment. Arch Insect Biochem Physiol 7: 29-36.

Hales, D.F., Tomiuk, J., Wohrmann, K. and Sunnucks, P. (1997) Evolutionary and genetic aspects of aphid 
biology: A review. Eur J Entomol 94: 1-55.

Hardie, J. (1981) Juvenile hormone and photoperiodically controlled polymorphism in Aphis fabae: prenatal effects on presumptive oviparae. J Insect Physiol 27: 257-265.

Hardie, J., Baker, F.C., Jamieson, G., Lees, A.D. and Schooley, D.A. (1985) The identification of an aphid juvenile hormone, and its titre in relation to photoperiod. Physiol Entomol 10: 297-302.

Hardie, J. and Lees, A.D. (1985) The induction of normal and teratoid viviparae by a juvenile hormone and kinoprene in two species of aphids. Physiol Entomol 10: 65-74.

Hardie, J. and Lees, A.D. (1985) Endocrine Control of Polymorphism and Polyphenism. In Comprehensive Insect Physiology Biochemistry and Pharmacology. (Kerkut, G.A., and Gilbert, L.I., eds.), Pergamon Press, Oxford, UK.

Hardie, J. (1987) The corpus allatum, neurosecretion and photoperiodically controlled polymorphism in an aphid. J Insect Physiol 33: 201-205.

Hartfelder, K. and Emlen, D.J. (2005) Endocrine Control of Insect Polyphenism. In Comprehensive Molecular Insect Science. (Gilbert, L.I., latrou, K., and Ganassi, S., eds.), pp. 651-703. Elsevier, Oxford, UK.

Huang, Z.-Y. and Robinson, G.E. (1995) Seasonal changes in juvenile hormone titers and rates of biosynthesis in honey bees. J Comp Physiol B 165: 18-28.

Huybrechts, J., Bonhomme, J., Minoli, S., Prunier-Leterme, N., Dombrovsky, A., Abdel-Latief, M., et al (2010) Neuropeptide and neurohormone precursors in the pea aphid, Acyrthosiphon pisum. Insect Mol Biol 19: 87-95.

International Aphid Genomics Consortium (2010) Genome Sequence of the Pea Aphid Acyrthosiphon pisum. PLoS Biol 8: e1000313.

Ishikawa, A., Hongo, S. and Miura, T. (2008) Morphological and histological examination of polyphenic wing formation in the pea aphid Acyrthosiphon pisum (Hemiptera, Hexapoda). Zoomorphology 
Kanbe, T. and Akimoto, S. (2009) Allelic and genotypic diversity in long-term asexual populations of the pea aphid, Acyrthosiphon pisum in comparison with sexual populations. Mol Ecol 18: 801-816.

Larkin, M.A., Blackshields, G., Brown, N.P., McGettigan, P.A., McWilliam, H., Valentin, F., et al (2007) Clustal W and Clustal X version 2.0. Bioinformatics 23: 2947-2948.

Le Trionnaire, G., Jaubert, S., Sabater-Munoz, B., Benedetto, A., Bonhomme, J., Prunier-Leterme, N., et al (2007) Seasonal photoperiodism regulates the expression of cuticular and signalling protein genes in the pea aphid. Insect Biochem Mol Biol 37: 1094-1102.

Le Trionnaire, G., Hardie, J., Jaubert-Possamai, S., Simon, J.-C. and Tagu, D. (2008) Shifting from clonal to sexual reproduction in aphids: physiological and developmental aspects. Biol Cell 100: 441-451.

Le Trionnaire, G., Francis, F., Jaubert-Possamai, S., Bonhomme, J., De Pauw, E., Gauthier, J.-P., et al (2009) Transcriptomic and proteomic analyses of seasonal photoperiodism in the pea aphid. BMC Genomics 10: 456.

Lees, A.D. (1959) The role of photoperiod and temperature in the determination of parthenogenetic and sexual forms in the aphid Megoura viciae Buckton - I. The influence of these factors on apterous virginoparae and their progeny. J Insect Physio/ 3: 92-117.

Lees, A.D. (1963) The role of photoperiod and temperature in the determination of parthenogenetic and sexual forms in the aphid Megoura viciae Buckton - III. Furthere properties of the maternal switching mechanism in apterous aphids. J Insect Physio/ 9: 153-164.

Lees, A.D. (1964) The location of the photoperiodic receptors in the aphid Megoura viciae Buckton. $J$ Exp Biol 41: 119-133.

Lees, A.D. (1966) The control of polymorphism in aphids. Adv Insect Physio/ 3: 207-277.

Lees, A.D. (1973) Photoperiiodic time measurement in the aphid Megoura viciae. J Insect Physiol 19: 2279-2316. 
Lees, A.D. (1981) Action spectra for the photoperiodic control of polymorphism in the aphid Megoura viciae. J Insect Physiol 27: 761-771.

Liu, Y., Henderson, G., Mao, L. and Laine, R.A. (2005) Seasonal Variation of Juvenile Hormone Titers if the Formosan Subterranean Termite, Coptotermes formosanus (Rhinotermitidae). Physiol Ecol 34: $557-562$.

Lungchukiet, P., Zhang, J., Tobe, S.S. and Bendena, W.G. (2008) Quantification of allatostatin receptor mRNA levels in the cockroach, Diploptera punctata, using real-time PCR. J Insect Physiol 54: 981-987.

Lungchukiet, P., Comeron Donly, B., Zhang, J., Tobe, S.S. and Bendena, W.G. (2008) Molecular cloning and characterization of an allatostatin-like receptor in the cockroach Diploptera punctata. Peptides 29: $276-285$.

Marcovitch, S. (1924) The migration of the aphididae and the appearance of the sexual forms as affected by the relative length of daily light exposure. J Agr Res 27: 513-522.

Mittler, T.E., Nassar, S.G. and Staal, G.B. (1976) Wing development and parthenogenesis induced in progenies of kinoprene-treated gynoparae of Aphis favae and Myzus persicae. J Insect Physiol 22: 1717-1725.

Mittler, T.E., Eisenbach, J., Searle, J.B., Matsuka, M. and Nassar, S.G. (1979) Inhibition by kinoprene of photoperiod-induced male production by apterous and alate viviparae of the aphid Myzus persicae. J Insect Physiol 25: 219-226.

Miura, T., Braendle, C., Shingleton, A., Sisk, G., Kambhampati, S. and Stern, D.L. (2003) A comparison of parthenogenetic and sexual embryogenesis of the pea aphid Acyrthosiphon pisum (Hemiptera: Aphidoidae). J Exp Zool 295B: 59-81.

Miura, T. (2005) Developmental regulation of caste-specific characters in social-insect polyphenism. Evol Dev 7: 122-129. 
Miyazaki, M. (1987) Forms and morphs of aphids. In Aphids, Their Biology, Natural Enemies and Control. (Minks, A.K., and Harrewijn, P., eds.), pp. 27-50. Elsevier Science Ltd, Amsterdam, NL.

Müller, C.B., Williams, I.S. and Hardie, J. (2001) The role of nutrition, crowding and interspecific interactions in the development of winged aphids. Ecol Entomol 26: 330-340.

Nijhout, H.F. and Wheeler, D.E. (1982) Juvenile hormone and the physiological basis of insect polymorphisms. Q Rev Biol 57: 109-133.

Nijhout, H.F. (1994) Insect Hormones. Princeton University Press, Princeton, US.

Nijhout, H.F. (1999) Control mechanisms of polyphenic development in insects - In polyphenic development, environmental factors alter same aspects of development in an orderly and predictable way. Bioscience 49: 181-192.

Nijhout, H.F. (2003) Development and evolution of adaptive polyphenisms. Evol Dev 5: 9-18.

Ollivier, M., Legeai, F. and Rispe, C. (2010) Comparative analysis of the Acyrthosiphon pisum genome and expressed sequence tag-based gene sets from other aphid species. Insect Mol Biol 19: 33-45.

Ramsey, J.S., Rider, D.S., Walsh, T.K., De Vos, M., Gordon, K.H.J., Ponnala, L., Macmil, S.L., Roe, B.A., and Janger, G. (2010) Comparative analysis of detoxification enzymes in Acyrthosiphon pisum and Myzus persicae. Insect Mol Biol 19: 155-164.

Sameshima, S.Y., Miura, T. and Matsumoto, T. (2004) Wing disc development during caste differentiation in the ant Pheidole megacephala (Hymenoptera : Formicidae). Evol Dev 6: 336-341.

Schwartzberg, E.G., Kunert, G., Westerlund, S.A., Hoffmann, K.H. and Weissera, W.W. (2008) Juvenile hormone titres and winged offspring production do not correlate in the pea aphid, Acyrthosiphon pisum. J Insect Physiol 54: 1332-1336.

Secher, T., Lenx, C., Cazzamali, G., Sorensen, G., Williamson, M., Hansen, G.N., et al (2001) Molecular Cloning of a Functional Allatostatin Gut/Brain Receptor and an Allatostatin Preprohormone from the Silkworm Bombyx mori. J Biol Chem 276: 47052-47060. 
Simon, J.-C., Rispe, C. and Sunnucks, P. (2002) Ecology and evolution of sex in aphids. Trends Ecol Evol 17: 34-39.

Stay, B. and Tobe, S.S. (2007) The role of allatostatins in juvenile hormone synthesis in insects and crustaceans. Annu Rev Entomol 52: 277-299.

Steel, C.G. and Lees, A.D. (1977) The role of neurosecretion in the photoperiodic control of polymorphism in the aphid Megoura viciae. J Exp Biol 67: 117-135.

Steel, C.G. (1978) Some functions of identified neurosecretory cells in the brain of the aphid, Megoura viciae. Gen Comp Endocrinol 34: 219-228.

Stern, D.L. and Foster, W.A. (1996) The evolution of soldiers in aphids. Biol Rev Cambridge Philos Soc 71: 27-79.

Tagu, D., Sabater-Munoz, B. and Simon, J.C. (2005) Deciphering reproductive polyphenism in aphids. Invertebrate Reprod Dev 48: 71-80.

Tanaka, S. (2001) Endocrine Mechanisms Controlling Body-Color Polymorphism in Locusts. Arch Insect Biochem Physiol 47: 139-149.

Vandesompele, J., De Preter, K., Pattyn, F., Poppe, B., Van Roy, N., De Paepe, A. and Speleman, F. (2002) Accurate normalization of real-time quantitative RT-PCR data by geometric averaging of multiple internal control genes. Genome Bio/ 3: research0034.0031-0034.0011.

Weaver, R.J. and Audsley, N. (2009) Neuropeptide Regulators of Juvenile Hormone Synthesis Structure, Functions, Distribution, and Unansered Questions. Trends Comp Endocrinol Neurobiol 1163: 316-329.

West-Eberhard, M.J. (2003) Developmental plasticity and evolution. Oxford University Press, Oxford, UK. Westerlund, S.A. and Hoffmann, K.H. (2004) Rapid quantification of juvenile hormones and their metabolites in insect haemolymph by liquid chromatography-mass spectrometry (LC-MS). Anal Bioanal Chem 379: 540-543. 
Wilkinson, T.L. and Ishikawa, H. (2000) Injection of essential amino acids substitutes for bacterial supply in aposymbiotic pea aphids (Acyrthosiphon pisum). Entomol Exp App/ 94: 85-91.

Zera, A.J. and Denno, R.F. (1997) Physiology and ecology of dispersal polymorphism in insects. Annu Rev Entomol 42: 207-230.

Zhou, X., Tarver, M.R. and Scharf, M.E. (2007) Hexamerin-based regulation of juvenile hormone-dependent gene expression underlies phenotypic plasticity in a social insect. Development 134: 601-610. 
Ishikawa et al.

JH-related genes

\section{Figures}

Figure 1 - The annual life cycle of the pea aphid

In spring, Foundress females (F-P), which hatch from the overwintering egg, start to produce their clonal daughters through viviparous parthenogenesis. They continue parthenogenesis during summer. In the parthenogenetic generation, parthenogenetic females produce winged $[\mathrm{P}-\uparrow(\mathrm{WD})]$ and wingless morphs [P-P (WL)] depending on their environment, including factors such as population density and host-plant quality. At the onset of fall, they switch the reproductive mode from viviparous parthenogenesis to sexual reproduction, i.e. male $(\widehat{\delta})$ and sexual oviparous female (S- + ) are produced and mate on their host plant. Sexual females lay overwintering eggs. Wing morphs in male $[\hat{\sigma}(\mathrm{WD})$ and $\hat{\alpha}(\mathrm{WL})]$ are determined genetically.

\section{Figure 2 - Reproductive schedules of aphids under long- or short-day lengths in the ApL strain}

(A) Mean number and standard errors of sexual or asexual morphs produced by aphids in the first generation under short-days (SG1, $n=8$ ). (B) Mean number and standard errors of morphs produced in the second generation under short-day lengths ( $S G 2, n=12)$.

Figure 3 - Protocol for rearing the pea aphids under long-days (16L/8D) or short-days (8L/16D) in the ApL strain

Under the long-days, only parthenogenetic females are constantly produced (LG1). In the first generation reared under short-days, parthenogenetic females are produced for about first 10 days, and then males are produced (SG1). In the second generation, oviparous sexual females are produced for about the first 10 days. After that, parthenogenetic females and males are produced (SG2). Gray boxes show the aphid sampling points. 
Ishikawa et al.

JH-related genes

Figure 4 - JH III titers in aphids producing sexual or asexual morphs in response to day-length conditions

Averages and standard errors are indicated $(n=5)$. In comparison with the aphids in parthenogenetic generations under the long-day conditions (LG1), significantly lower $\mathrm{JH}$ titers were detected in the aphids under short-days (SG1 and SG2). The different letters on bars denote significant differences among categories (Kruskal-Wallis test followed by the Peritz test, $\mathrm{p}<0.05$ )

Figure 5 - Expression profiles of $\mathrm{JH}$ related genes under long- and short-day conditions

The Y-axes show relative expression levels, which are specific to each gene. Averages and standard errors of three biological replicates are indicated. The different letters on the bars denote significant differences among categories (one-way ANOVA followed by the Tukey-Kramer test, $p<0.05$ ).

\section{Figure 6 - Correlation between JHIII titer and the expression of JHE1}

The X-axes show JHIII titer and Y-axes show the relative expression of JHE1. Averages and standard errors are indicated. JHIII titer and the expression level of JHE1 are negatively and strongly correlated (Speaman's rank correlation, $r s=-0.89, \mathrm{p}<0.05)$. 
Table 1. List of JH-related genes in the pea aphid

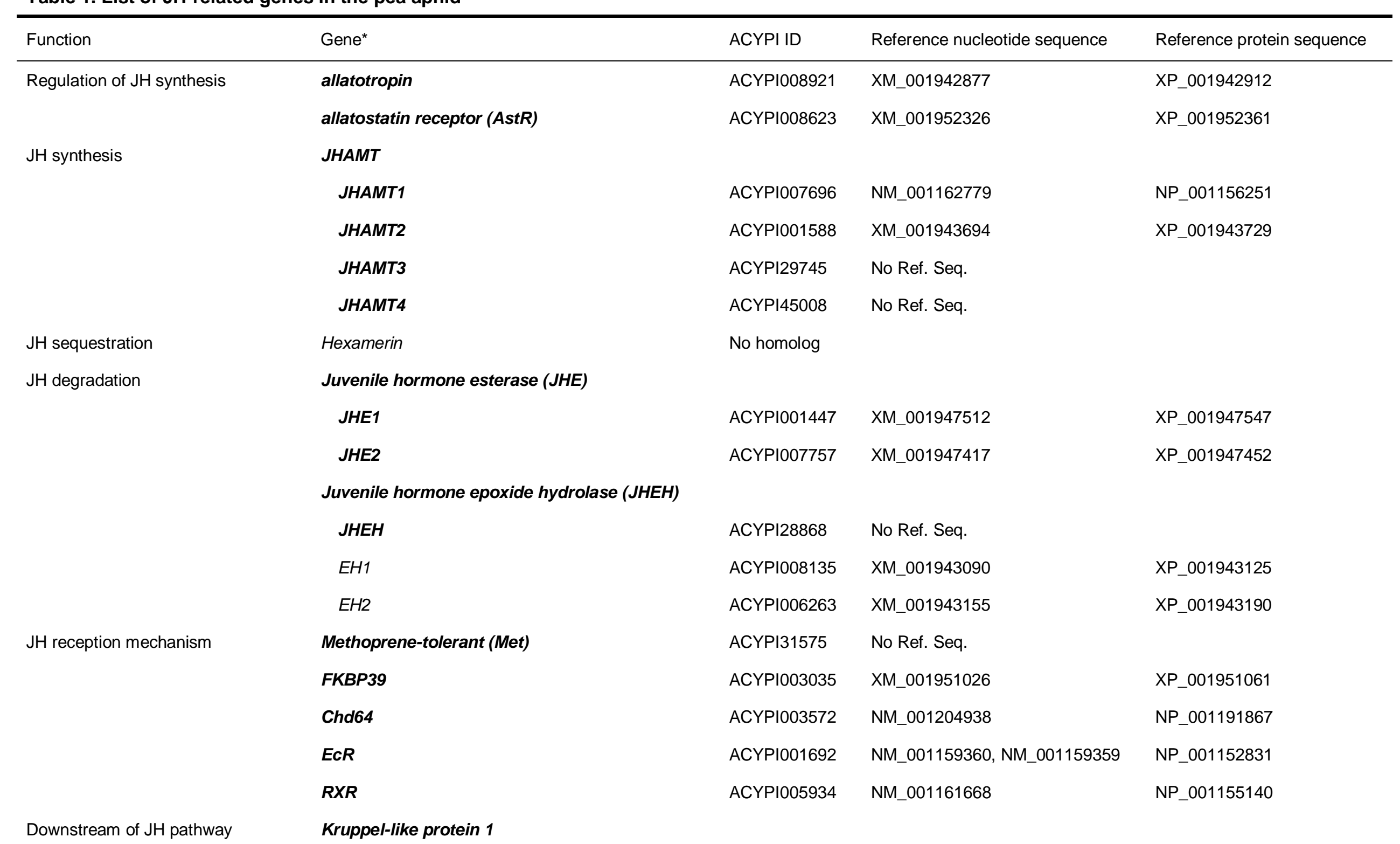


Ishikawa et al.

Kr-h1 1

Kr-h1 2

Downstream of Ecdysone pathway

broad

*Genes in bold text are those used for gene expression level anaysis in this study
$\mathrm{JH}$-related genes

ACYPI000598

XM 001946159

XP_001946194

ACYPI006236

XM_001944388

XP_001944423

ACYPI008576

XP_001942681 


\section{Supporting information}

Figure S1 - Phylogenetic tree of Allatostatin Receptors.

Figure S2 - Phylogenetic tree of PPOs and Hexamerins.

Figure S3 - Phylogenetic tree of Epoxide hydrolases.

Figure S4 - Phylogenetic tree of Met.

Figure S5 - Phylogenetic tree of FKBP39.

Figure S6 - Phylogenetic tree of Chd64.

Figure S7 - Phylogenetic tree of Kr-h1s.

Figure S8 - Phylogenetic tree of Broad.

Table S1 - List of primer sequences for qRT-PCR. 


\section{Parthenogenesis}

\section{Summer}

\section{Spring}

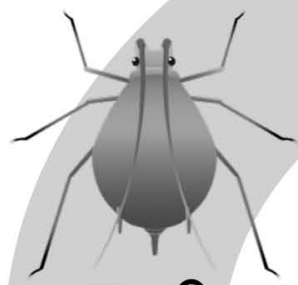

$$
\text { F- 우 }
$$

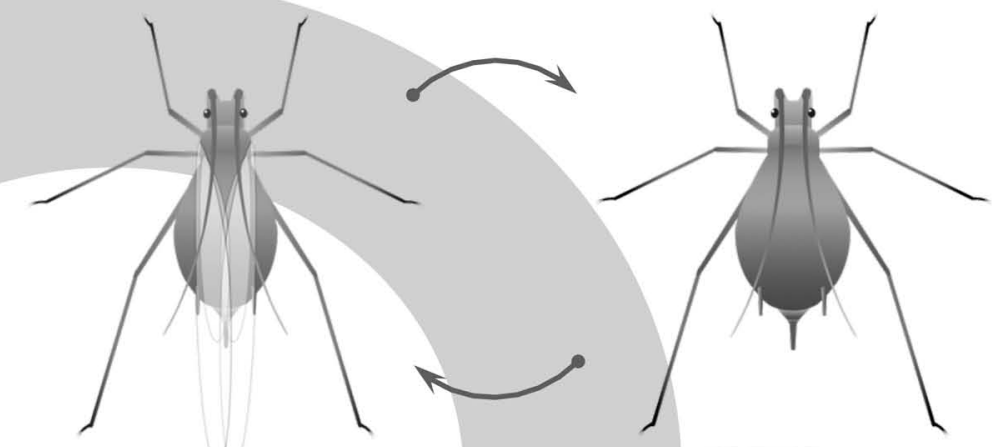

\section{Winter}

$$
\text { P-우(WD) }
$$

$$
\text { P-오 (WL) }
$$

\section{8}
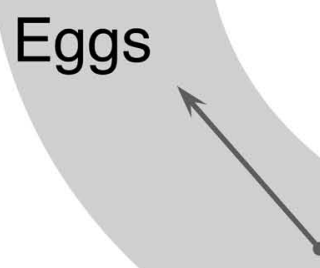

Autumn

\section{Sexual reproduction}




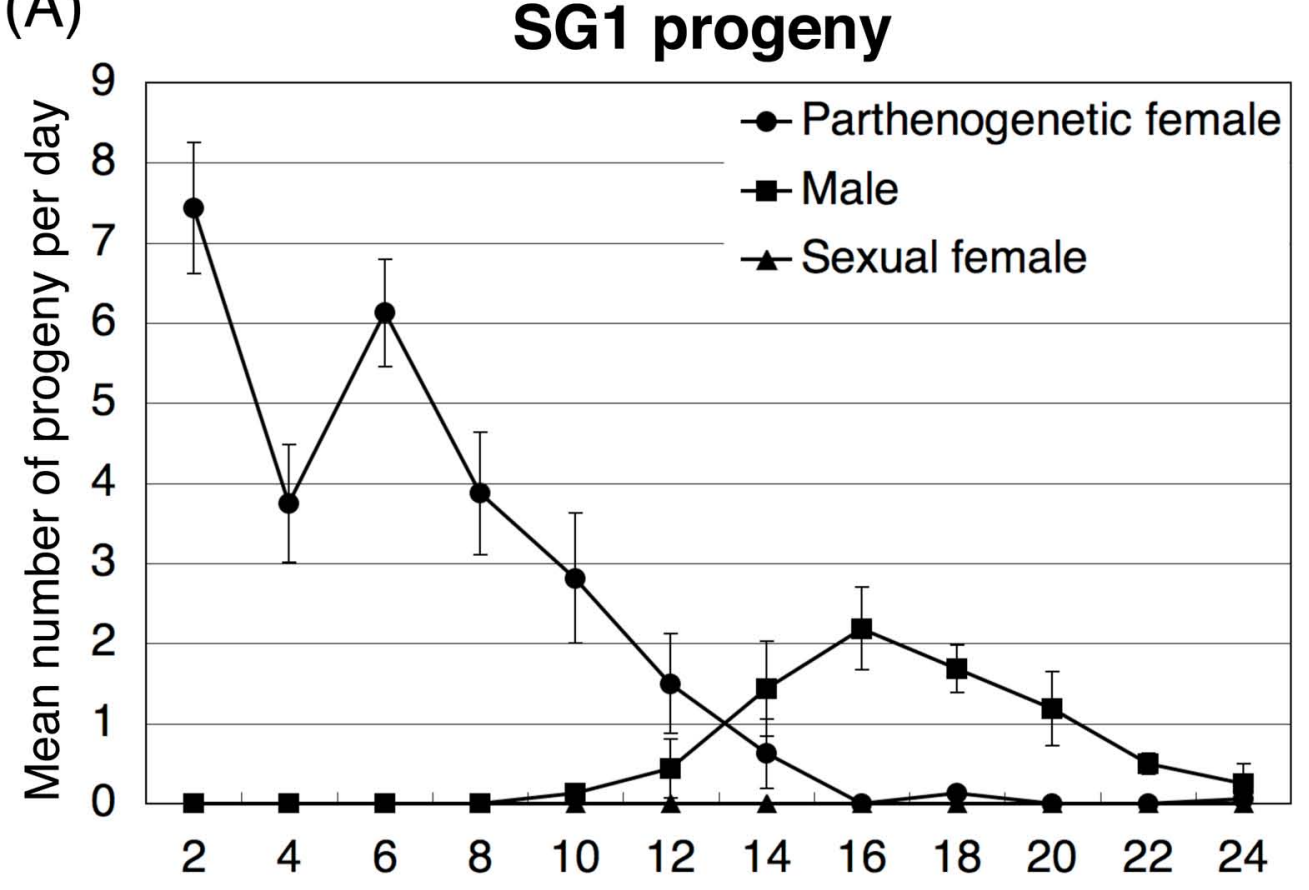

Days after first larviposition

(B)

SG2 progeny

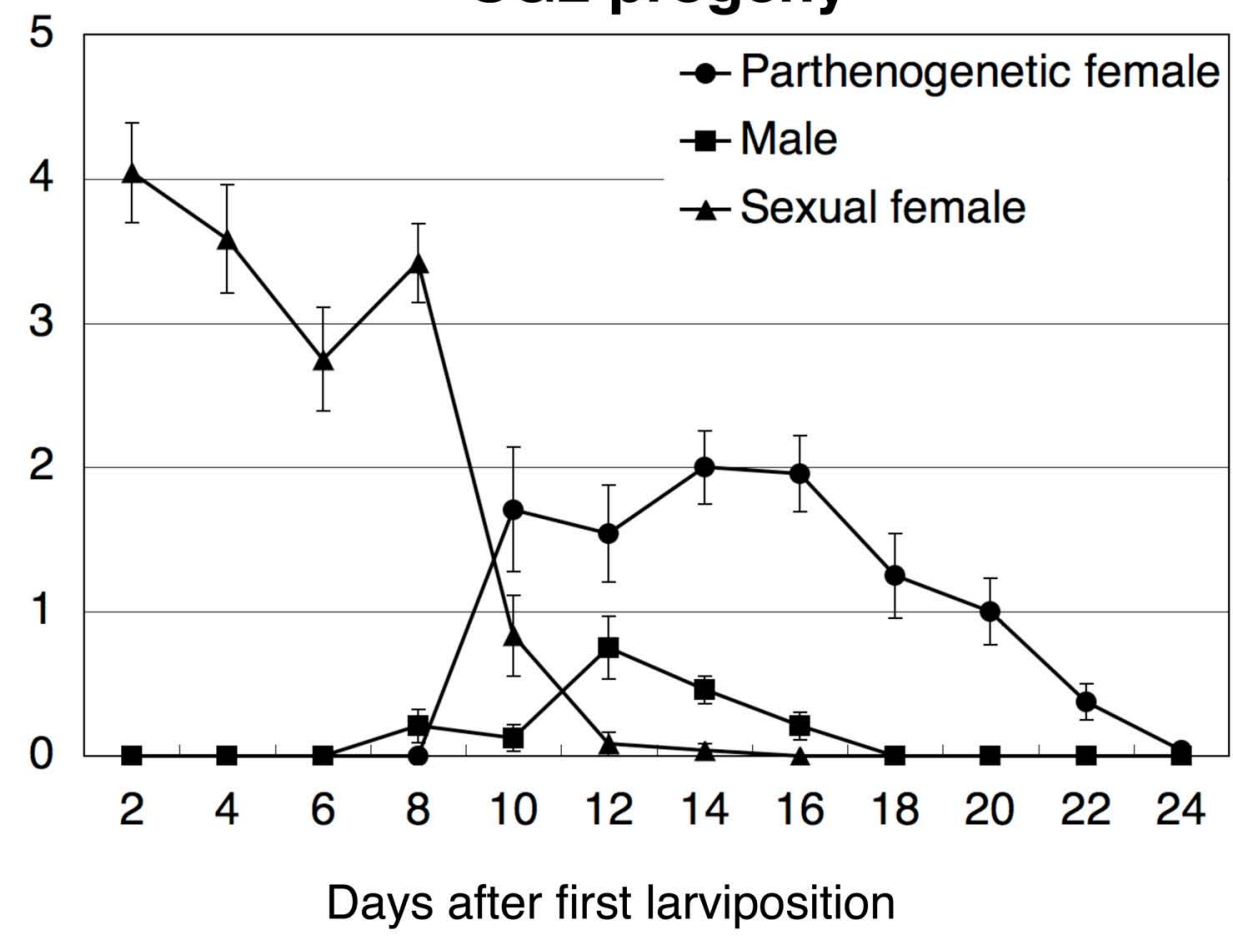




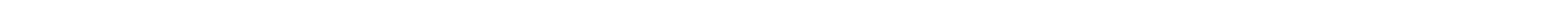




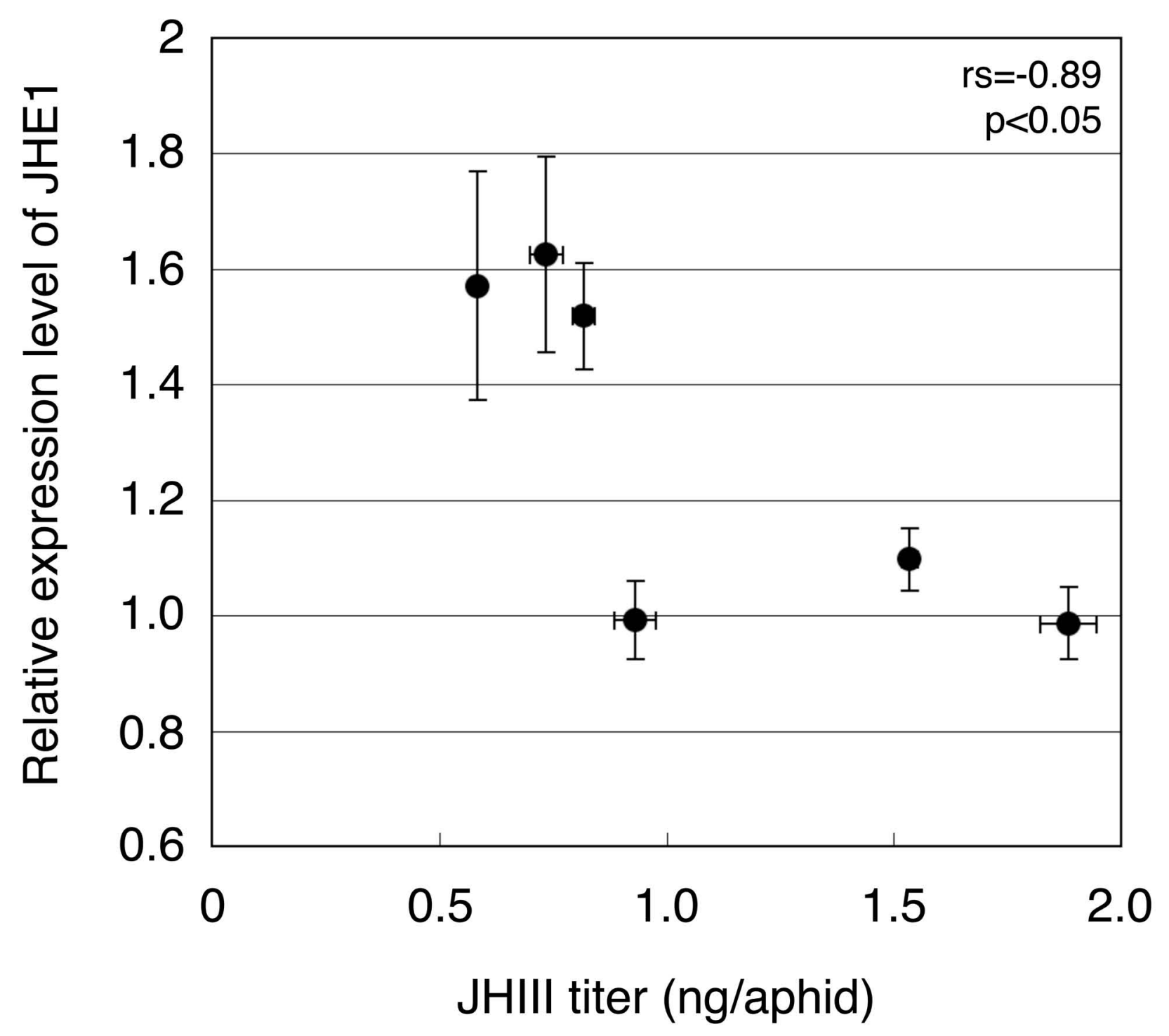

